\title{
Left Ventricular Outflow Tract Migration of a Balloon-Expandable Prosthesis During Transcatheter Aortic Valve Implantation
}

\author{
Transcatheter Bailout Is Feasible, but not Safe \\ Daisuke Hachinohe, ${ }^{1}$ MD, Ken Kobayashi, ${ }_{1}^{1}$ MD, Azusa Furugen, ${ }^{1}$ MD, \\ and Ryuji Koshima, ${ }^{1} \mathrm{MD}$
}

\begin{abstract}
SUMMARY
Valve migration into the left ventricular outflow tract (LVOT) during transcatheter aortic valve implantation (TAVI) is a life-threatening complication. An 89-year-old female patient was admitted for TAVI due to severe symptomatic aortic stenosis. After deployment of a balloon-expandable prosthesis, the prosthesis had migrated into the LVOT. The prosthesis was reimpacted to the aortic annulus by a balloon-assisted recapture procedure. Immediately after recapturing the prosthesis with an oversized balloon, the patient's vital signs deteriorated due to acute aortic regurgitation (AR), and a prompt valve-in-valve ( $\mathrm{V}$-in-V) procedure allowed us to stabilize the patient's condition. This is the first reported case of a $\mathrm{V}$-in-V procedure using an oversized balloon and a larger prosthesis to treat migration of the initial prosthesis into the LVOT. Balloon recapture and V-in-V procedure using an oversized balloon and larger prosthesis for a migrated balloonexpandable prosthesis into the LVOT is feasible, but hemodynamic support should be prepared before recapture and Vin-V because overdilatation of the first prosthesis might cause hemodynamic collapse due to severe AR. (Int Heart J 2017; 58: 290-293)
\end{abstract}

Key words: Aortic stenosis, Prosthesis embolization, TAVI, TAVR, TVE, Valve-in-valve

$\mathrm{M}$ ore than $30 \%$ of geriatric patients with symptomatic severe heart valve disease are treated conservatively. ${ }^{1)}$ Transcatheter aortic valve implantation (TAVI) is an alternative treatment for aortic stenosis (AS) in patients for whom surgery is extremely risky or contraindicated. ${ }^{2)} \mathrm{Al}-$ though evolving device technology and greater experience are associated with improved outcomes, procedural complications are still common. Valve embolization into the left ventricle (LV) is a life-threatening complication that requires prompt diagnosis and treatment. ${ }^{3)}$ Here, we present a case of a rare but serious complication in which a balloon-expandable prosthesis (SAPIEN XT, Edwards Lifesciences, USA) migrated into the left ventricular outflow tract (LVOT). The prosthesis was successfully reimpacted to the aortic annulus by balloon-assisted recapture and a valve-in-valve $(\mathrm{V}$-in- $\mathrm{V})$ procedure using femoral access without surgical conversion or the occurrence of any sequelae.

\section{CASE Report}

An 89-year-old female patient was referred to our hospital for treatment for severe symptomatic AS. She had NYHA class-III symptoms, which were refractory to optimal medical therapy. Transthoracic echocardiography revealed severe AS with an aortic valve area (AVA) of $0.77 \mathrm{~cm}^{2}$, an AVA index of $0.60 \mathrm{~cm}^{2} / \mathrm{m}^{2}$, and a preserved ejection fraction of $66 \%$. Her annular area was $385 \mathrm{~mm}^{2}$ on computed tomography (CT). The non-coronary and right-coronary leaflets had a severe amount of calcification, but the left-coronary cusp lacked significant calcification (Figure 1). The patient was referred for TAVI because she was considered to be at a high risk by our multidisciplinary heart team due to her logistic EuroSCORE of 25.28\%, Society of Thoracic Surgeons score of $6.152 \%$, and significant frailty. A transfemoral approach was selected because of her anatomic features and the low invasiveness.

The patient underwent TAVI under general anesthesia. Because of the borderline annulus size, a calibrated balloon aortic valvuloplasty (BAV) was performed during rapid right ventricular pacing. Aortic root angiography during BAV showed that the 20-mm balloon completely occluded the annulus, and no contrast backflow into the LV was seen. In addition, the calculated annulus size measured on CT by 6 welltrained physicians using Ziostation2 (Ziosoft Inc., Tokyo) was $385 \mathrm{~mm}^{2}$ on average, so a 23-mm SAPIEN XT prosthesis was chosen. Although the prosthesis had been properly positioned just before the deployment (Figure 2), rapid ventricular pacing failed at the last moment of deployment.

Ten minutes after deployment, fluoroscopy demonstrated that the prosthesis had migrated to the LVOT (Figure 3). We

From the ${ }^{1}$ The Sapporo Heart Center, Sapporo Cardiovascular Clinic, Sapporo, Japan.

Address for correspondence: Daisuke Hachinohe, MD 8-1, Kita-49 Higashi-16, Higashiku, Sapporo, Hokkaido 007-0849 Japan. Email: heartbond.8@ gmail.com Received for publication June 18, 2016. Revised and accepted July 20, 2016.

Released in advance online on J-STAGE March 17, 2017.

All rights reserved by the International Heart Journal Association. 


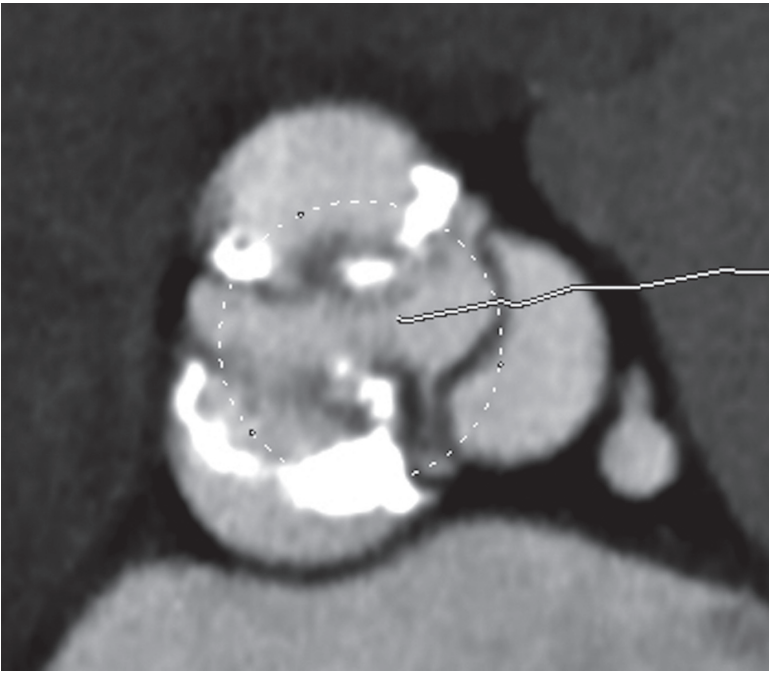

Figure 1. Asymmetric calcification of the native aortic valve. The noncoronary and right-coronary leaflets had a severe amount of calcification, but the left-coronary cusp lacked significant calcification.

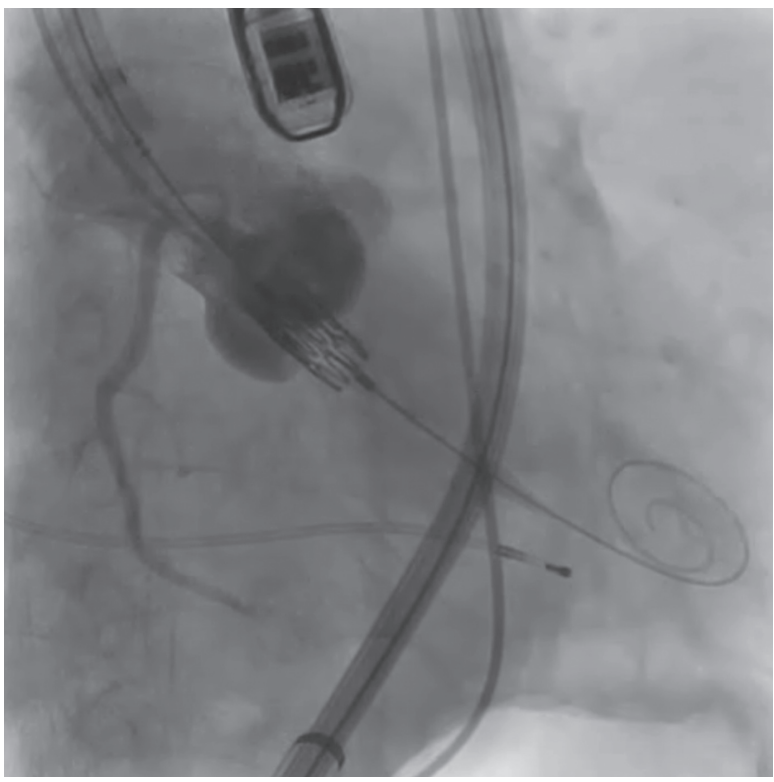

Figure 2. Position of prosthesis was good just before the deployment.

decided to recapture the migrated prosthesis and reimpact it into the aortic annulus. Unfortunately, as the Safari wire (Boston Scientific, USA) was already removed from the LV, we managed to cross a Radifocus guidewire (Terumo, Japan) with a 4-Fr AL1.0 catheter (Terumo) into the LV through both the native aortic valve and the prosthesis. Finally, the wire could pass into the LV and was successfully changed to a Safari wire using a 5-Fr pigtail catheter (Terumo). Immediately after placing the Safari wire in the apical LV, the migrated prosthesis fell down from the LVOT into the LV. The embolized prosthesis was retracted with a $23-\mathrm{mm}$ balloon and repositioned at the annulus during rapid pacing, but the prosthesis embolized into the LV despite a few attempts to retrieve it. We then recognized that the 23-mm prosthesis was undersized and decided to use

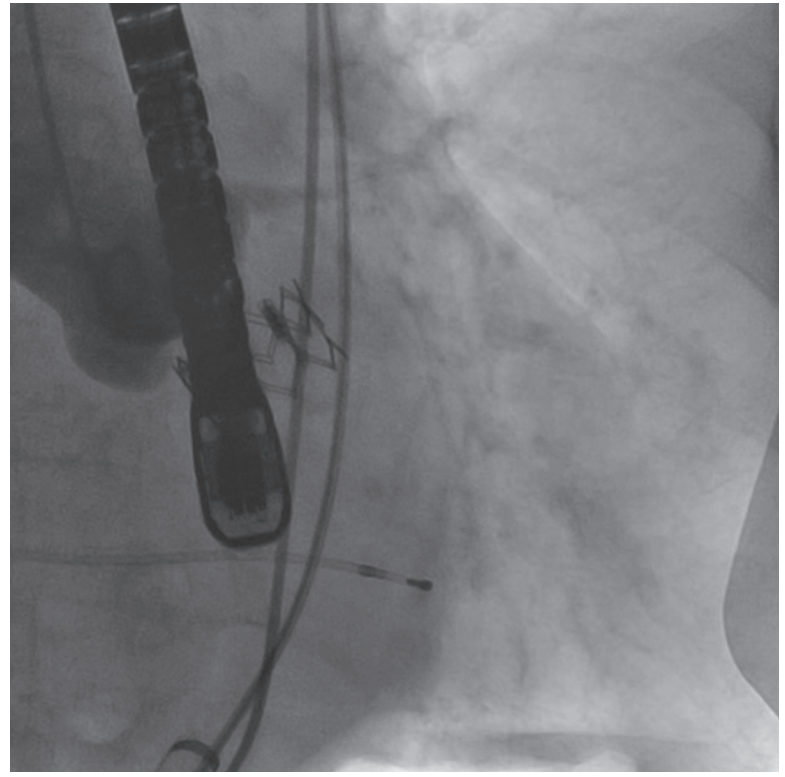

Figure 3. Migration of the implanted prosthesis. The implanted prosthesis had migrated to the left ventricle ventricular outflow tract.

an oversized balloon to reimpact the prosthesis into the annulus and perform a V-in-V procedure using a 26-mm SAPIEN $\mathrm{XT}$ prosthesis. The embolized $23-\mathrm{mm}$ prosthesis was pulled up using a 25-mm balloon with underexpansion and impacted into the annulus with nominal pressure (Figure 4A, Figure 4B). Immediately after full inflation, the patient fell into cardiopulmonary arrest. After a few minutes of resuscitation with pharmacological agents and heart massage, her vital signs improved but remained imperfect. We quickly changed the eSheath (Edwards Lifesciences, USA) from 16-Fr to 18-Fr and implanted a second 26- $\mathrm{mm}$ prosthesis with $1 \mathrm{~mL}$ lower volume within the first 23-mm prosthesis at a slightly higher position (Figure 5A, Figure 5B). After the $\mathrm{V}$-in-V procedure, the patient promptly regained hemodynamic stability. Transesophageal echocardiography (TEE) and aortography revealed neither significant aortic regurgitation (AR) nor other potential complications such as annulus rupture or LV perforation.

\section{Discussion}

Here, we have reported a prosthesis that migrated into the LVOT and was successfully recaptured and replaced with a larger prosthesis via the femoral artery. Immediately after recapturing the prosthesis with an oversized balloon, the patient's vital signs deteriorated due to acute $\mathrm{AR}$, and a prompt $\mathrm{V}$-in-V procedure allowed us to stabilize her condition.

Despite the fact that TAVI is less invasive than surgical aortic valve replacement, periprocedural complications are still common. Among these, prosthesis migration into the LV is a rare but catastrophic complication and requires emergency surgical conversion, which is associated with high mortality rates ${ }^{3,4)}$

Several factors are associated with prosthesis migration, and in most cases the precipitating factor can be identified. ${ }^{5-7)}$ In this case, there were a number of possible causes. One pos- 

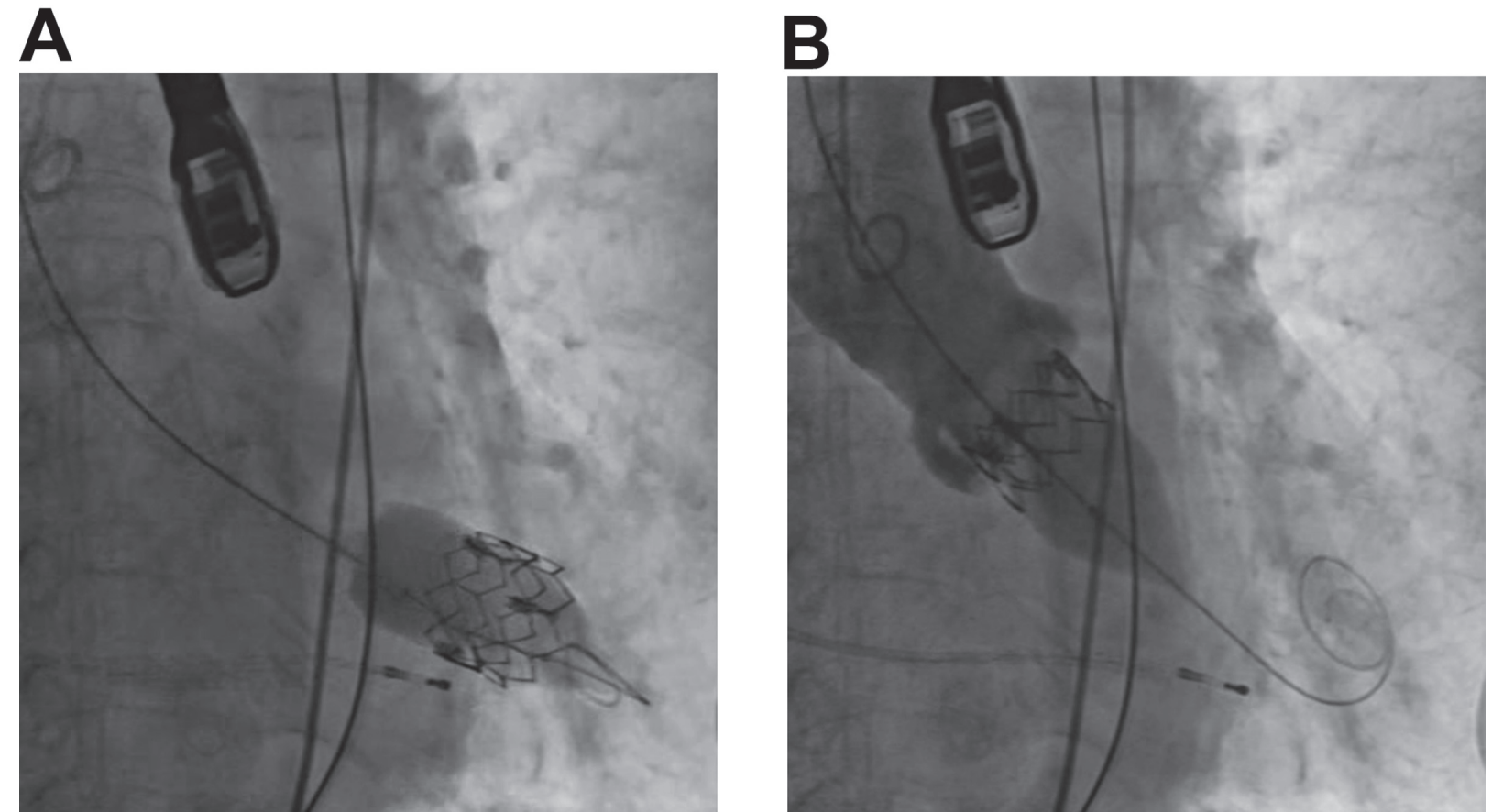

Figure 4. Retraction and reimpaction using an oversized balloon. A: Retraction of the embolized prosthesis with a 25-mm balloon. B: Repositioning at the annulus.
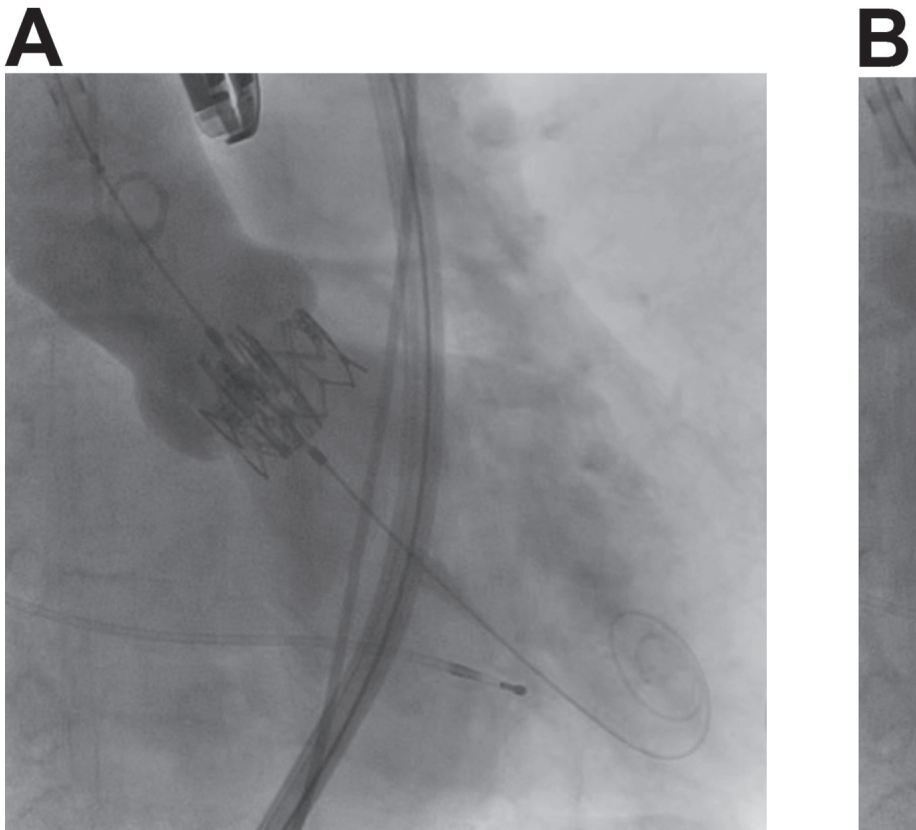

Figure 5. Valve-in-valve procedure. A: A 26-mm prosthesis at a slightly higher position. B: Valve-in-valve was successfully performed.

sible cause was asymmetric calcification of the native aortic valve. An asymmetrically calcified aortic valve might prevent the prosthesis from becoming coaxial position to the annular plane leading to prosthesis instability. As matter of fact, while there are severe calcifications in non-coronary and right-coronary leaflets, left-coronary leaflets had no significant calcification (Figure 1). Another possible cause was the use of an inap- propriately sized prosthesis. Accurate measurement of the annulus by CT and TEE is very important. ${ }^{8)}$ In the present case, the measurements reported by $\mathrm{CT}$ and TEE were 385 $\mathrm{mm}^{2}$ and $353 \mathrm{~mm}^{2}$, respectively. In addition, a calibrated BAV with contrast injection at the aortic root showed no backflow into the LV, so we chose to use a 23-mm prosthesis. However, movement of the balloon could be seen during BAV, which 
may have been suggestive of insufficient coverage of the annulus. This implied that a larger prosthesis should have been chosen. During calibrated BAV, assessment of contrast backflow into the LV is less important than the anatomical relationship between the sinus hinge points and the balloon. ${ }^{9)}$ Another important cause of prosthesis migration in the present case was pacemaker malfunction during implantation. Premature termination of rapid pacing before full expansion of the prosthesis causes non-coaxiality to the annular plane, leading to prosthesis migration. ${ }^{4,70)}$ Proper pacing should be able to prevent such occurrences. To prevent prosthesis migration, careful preprocedural planning with precise assessment of the aortic root by CT and TEE should be performed. In addition, contrast backflow into the LV during calibrated BAV should not be assessed as a major criterion but considered alongside other evidence.

How should we treat when it occurs? Embolization into the aorta can sometimes be treated by valve repositioning and deploying into the distal aorta using transcatheter techniques with good outcomes on mid-term to long-term follow-up. ${ }^{5,7,11)}$ Meanwhile, migration of prostheses into the LV can be more challenging, and in such cases, immediate conversion to surgical treatment is often required. ${ }^{5,10)}$ To the best of our knowledge, only 4 cases have been reported which could be recovered using a transcatheter approach without conversion to surgery: two cases were resolved using the transapical approach, ${ }^{12,13)}$ and the others using the transfemoral approach. ${ }^{4,14)}$ According to these reports, after LV migration of the prosthesis, recapture and V-in-V technique using transcatheter techniques seem to be safe and feasible. Rescue V-in-V has the potential to avoid the need for emergency surgery and has come to play an important role in TAVI.

Most importantly, in all previously reported cases, the second prosthesis was the same size as the first. To the best of our knowledge, this is the first reported case of a $\mathrm{V}$-in- $\mathrm{V}$ procedure using a larger prosthesis to treat migration of the initial prosthesis into the LVOT. In such cases, oversized balloons might be needed to recapture an undersized migrated prosthesis and reimpact to the annulus, which can definitely cause hemodynamic collapse due to severe AR. Therefore, hemodynamic support such as extracorporeal membrane oxygenation should be prepared before recapturing the prosthesis using an oversized balloon.

Conclusion: This is the first reported case in which a migrated prosthesis into the LVOT was successfully bailed with an oversized balloon recapturing and a $\mathrm{V}$-in- $\mathrm{V}$ procedure using a larger prosthesis. This bailout technique is feasible, but hemodynamic support should be prepared before recapture and V-in-V because overdilatation of the first prosthesis might cause hemodynamic collapse due to severe AR.

\section{Disclosure}

The authors declare that there is no conflict of interest.

\section{ACKNOWLEDGMENTS}

This case was managed by Umihiko Kaneko, Takeshi Kawamura, MD, Satoshi Sumino, MD, Tsutomu Fujita, MD and Hirosato Doi, MD as a multidisciplinary heart team. We would like to express our gratitude to Shinichi Shirai, MD who participated in this case as a supervisory doctor. We would also like to offer our special thanks to our support medical staff.

\section{REFERENCES}

1. Hu K, Wan Y, Hong T, et al. Therapeutic decision-making for elderly patients with symptomatic severe valvular heart diseases. Int Heart J 2016; 57: 434-40.

2. Ayhan H, Durmaz T, Keleș T, et al. Simultaneously successful transfemoral aortic valve implantation and endovascular repair of thoracic aortic saccular aneurysm. Int Heart J 2014; 55: 459-62.

3. Hein R, Abdel-Wahab M, Sievert H, et al. Outcome of patients after emergency conversion from transcatheter aortic valve implantation to surgery. EuroIntervention 2013; 9: 446-51.

4. Seecheran N, Ittleman F, Dauerman H. Left ventricular outflow tract embolization and balloon assisted recapture of a SAPIEN XT prosthesis during transcatheter aortic valve replacement. Catheter Cardiovasc Interv 2016; 88: E89-92.

5. Ibebuogu UN, Giri S, Bolorunduro O, et al. Review of reported causes of device embolization following trans-catheter aortic valve implantation. Am J Cardiol 2015; 115: 1767-72. (Review)

6. Makkar RR, Jilaihawi H, Chakravarty T, et al. Determinants and outcomes of acute transcatheter valve-in-valve therapy or embolization: a study of multiple valve implants in the U.S. PARTNER trial (Placement of AoRTic TraNscathetER Valve Trial Edwards SAPIEN Transcatheter Heart Valve). J Am Coll Cardiol 2013; 62: 418-30.

7. Tay EL, Gurvitch R, Wijeysinghe N, et al. Outcome of patients after transcatheter aortic valve embolization. JACC Cardiovasc Interv 2011; 4: 228-34.

8. Jilaihawi H, Kashif M, Fontana G, et al. Cross-sectional computed tomographic assessment improves accuracy of aortic annular sizing for transcatheter aortic valve replacement and reduces the incidence of paravalvular aortic regurgitation. J Am Coll Cardiol 2012; 59: 1275-86.

9. Shivaraju A, Thilo C, Ott I, et al. Tools and Techniques - Clinical: Fluoroscopic balloon sizing of the aortic annulus before transcatheter aortic valve replacement (TAVR) - follow the "right cusp rule". EuroIntervention 2015; 11: 840-2.

10. Al Ali AM, Altwegg L, Horlick EM, et al. Prevention and management of transcatheter balloon-expandable aortic valve malposition. Catheter Cardiovasc Interv 2008; 72: 573-8.

11. Chakravarty $\mathrm{T}$, Jilaihawi $\mathrm{H}$, Doctor $\mathrm{N}$, et al. Complications after transfemoral transcatheter aortic valve replacement with a balloonexpandable prosthesis: The importance of preventative measures and contingency planning. Catheter Cardiovasc Interv 2013 (in press)

12. Dumonteil N, Marcheix B, Grunenwald E, Roncalli J, Massabuau P, Carrié D. Left ventricular embolization of an aortic balloon-expandable bioprosthesis: balloon capture and reimpaction as an alternative to emergent conversion to open-heart surgery. JACC Cardiovasc Interv 2013; 6: 308-10.

13. Čanádyová J, Mokráček A, Pešl L, Kurfirst V. How to manage left ventricular embolization of the transcatheter valve. Kardiochir Torakochirurgia Pol 2015; 12: 53-5.

14. Barbash IM, Bogdan A, Fefer P, et al. How should I treat a left ventricular outflow tract-migrated balloon-expandable transcatheter heart valve? EuroIntervention 2016; 11: 1442-5. 